\title{
Intermédialités
}

Histoire et théorie des arts, des lettres et des techniques

Intermediality

History and Theory of the Arts, Literature and Technologies

\section{L’imprimé et le non-imprimé}

\section{Théorie des médias et poétique du papier}

\section{Lothar Müller}

Numéro 17, printemps 2011

reproduire

reproducing

URI : https://id.erudit.org/iderudit/1005746ar

DOI : https://doi.org/10.7202/1005746ar

Aller au sommaire du numéro

\section{Éditeur(s)}

Revue intermédialités (Presses de l’Université de Montréal)

ISSN

1705-8546 (imprimé)

1920-3136 (numérique)

Découvrir la revue

Citer cet article

Müller, L. (2011). L’imprimé et le non-imprimé : théorie des médias et poétique du papier. Intermédialités / Intermediality, (17), 19-29.

https://doi.org/10.7202/1005746ar

\section{Résumé de l'article}

Cet article étudie selon une approche alternative l'histoire culturelle du papier dans l'Europe occidentale moderne, en remettant en perspective les interactions entre papier imprimé et papier non imprimé. On y trouve une critique de la formule diachronique de Marshall McLuhan : « du manuscrit à l'imprimé ». McLuhan soutient que l'invention de l'imprimerie a conduit à une transformation historique brutale : le papier non imprimé n'a plus servi qu'à nourrir la production de papier imprimé, tandis que le statut moderne de l'auteur s'est trouvé irrévocablement rattaché à la nouvelle matérialisation de l'innovation et de l'autorité : le caractère d'imprimerie. Cet article préfère mettre l'accent sur les différentes strates dans l'usage du papier, telles qu'on les découvre dans les pratiques culturelles de la première modernité européenne (on peut penser au papier comme médium visuel), et plaide pour une réinterprétation de l'impact produit par l'imprimerie. Imprimé et non-imprimé ont été considérés à égalité et sont restés inséparables, car ils étaient chacun l'effet de la demande croissante de papier dans tous les domaines de la vie publique et privée. Pour montrer les effets culturels de cette tension entre papier imprimé et non imprimé, l'article propose une analyse de la fiction de l'éditeur. On sait que dans certains textes poétiques, le produit imprimé se réfère à sa propre histoire, en remontant à un manuscrit sur papier non imprimé.
Ce document est protégé par la loi sur le droit d'auteur. L'utilisation des services d'Érudit (y compris la reproduction) est assujettie à sa politique d'utilisation que vous pouvez consulter en ligne.

https://apropos.erudit.org/fr/usagers/politique-dutilisation/ 


\title{
L’imprimé et le non-imprimé
}

\section{Théorie des médias et poétique du papier}

\author{
LOTHAR MÜLler
}

epuis le $15^{\mathrm{e}}$ siècle le papier a fusionné avec la presse d'imprimerie avec
un tel succès qu'il est avant tout perçu dans cette unité, aussi bien dans la conscience générale que dans les théories des médias. Il apparaît comme un élément passif, une matière molle sur laquelle se gravent les lettres de métal, contribuant ainsi à la circulation massive de l'écriture et à sa conservation. La vulgarisation de la théorie de la «galaxie Gutenberg» développée par Marshall McLuhan a contribué à renforcer la tendance qui consiste à appréhender le papier comme un carburant pour la presse d'imprimerie. Chez McLuhan, le papier alimente la dynamique de cette dernière et il attire surtout l'attention sur lui ex negativo - soit au moment où son réapprovisionnement fait défaut. Dans l'horizon de l'analogie avec le carburant, le papier fonctionne comme condition de possibilité de l'impression. C'est en tant que substance porteuse de téléologie qu'il entre dans la formation du typographic man et promeut une universalisation du code alphabétique à la mesure même de sa standardisation, de son uniformisation et de sa reproduction mécanique continuelle. Dans la «galaxie Gutenberg» de McLuhan, le papier est le support toujours prêt à recevoir l'écriture et l'image, le fond sur lequel se profile le triomphe de la linéarité et de l'abstraction marquant de leur sceau les mentalités, ainsi que la pétrification typographique et l'hypertrophie du visuel qui caractérisent l'ensemble de la culture des Temps modernes avant que les médias électroniques ne rompent le charme'.

Le papier n’est toutefois pas aussi complètement déterminé par la logique de la presse d'imprimerie que l'analogie avec le carburant le suggère. Comme c'est une substance non spécifique et que ce n'est ni une chose ni un outil, son succès historique provient moins de la fixation d'un but précis que de la versatilité avec laquelle il se prête à la réalisation de toutes sortes de buts. À partir du $13^{\mathrm{e}}$ siècle

1. Voir Herbert Marshall McLuhan, The Gutenberg Galaxy, Londres, Routledge, 1962 et Understanding Media: The Extensions of Man, New York, McGraw-Hill, 1964. 
et à commencer par le nord de l'Italie, les moulins à papier avec lesquels les Européens reprirent la technique de fabrication du papier utilisée par les Arabes, en l'intégrant à un commerce et une mécanisation alors en expansion, n'imposaient pas le poids de la concurrence au seul parchemin des scribes du Moyen Âge. Avant que la presse d'imprimerie ne se développe, ils approvisionnaient déjà les chancelleries et les commerçants dont les besoins en papier s'étaient accrus en raison des techniques de gestion telles que la tenue des livres de comptabilité en partie double. De plus, l'établissement de nouveaux moulins mobilisant du capital au nord comme au sud des Alpes ne répondait pas exclusivement à un accroissement des besoins en papier. Si le premier moulin à papier allemand qu'Ulman Stromer établit autour de 1390 à Nuremberg profita aussi des besoins en papier de l'administration communale et seigneuriale, il n'en écoulait pas moins une bien plus grande partie de sa production en tant qu'entreprise de soustraitance de cartes à jouer. Au $14^{\mathrm{e}}$ siècle, la dépendance vis-à-vis des cartes à jouer qui touchait aussi les couches illettrées de la population donna naissance, par la combinaison de la gravure et de la technologie du papier, à une première forme de médium de divertissement de masse qui laissa ses traces dans l'étymologie du papier dans les langues européennes. Les Italiens nommèrent Naibbe le jeu de hasard composé de cartes représentant les gouverneurs mamelouks qui tenaient le premier rôle dans les cartes égyptiennes. Ces premiers produits de masse en circulation sur du papier obtinrent leur nom de leur support: cartce. Le mot carta reçut par conséquent une signification double: il pouvait désigner une feuille de papier normale ou bien une carte à jouer, et c'est dans la deuxième acception qu'il migra à travers l'Europe, ainsi que dans les langues anglaise et française.

Le développement des entrepôts et des formats de papier auxquels les imprimeurs pouvaient recourir lors de l'impression de livres, d'affiches, de feuilles volantes et aussi de journaux à partir du $17^{\mathrm{e}}$ siècle, fit augmenter la quantité de papier non imprimé qui trouva son utilisation dans la conservation et la circulation de l'écriture et de l'image sur des distances différentes dans le temps et l'espace. Le papier contribua à la révolution médiatique qui eut lieu au début des Temps modernes par l'intermédiaire de la presse d'imprimerie. Cette révolution, dont l'orientation générale met en relief la formule from manuscript to print, trouve son équivalent dans cette infiltration plutôt neutre du papier non imprimé dans les capillaires du système juridique et des courants d'échanges commerciaux, de l'administration des communes et des résidences, sans oublier la scripturalisation de la vie privée. Que ce soit la comptabilité commerçante ou l'introspection et l'établissement continu du bilan de vie dans le journal intime qui gagnent du terrain, il se développe d'un côté des habitudes qui 
pérennisent l'approvisionnement en papier et de l'autre des techniques culturelles pour lesquelles ce participant silencieux devint essentiel. Une histoire du médium papier ne se laisse donc pas facilement réduire à la représentation courante de l'«ère Gutenberg» et, de son point de vue fixe, à la typographie ou, moins encore, au livre imprimé. Elle se doit moins de reconstituer les effets d'une invention spectaculaire que d'envisager la somme des constellations dans lesquelles un small tool fait office de condition de possibilité du développement de techniques culturelles.

Le papier est une matière première de l'époque industrielle qui prend racine au début des Temps modernes. C'en est une au même titre que le bois, la pierre et le métal, mais c'est aussi de plus en plus un produit de la civilisation: d'une part parce qu'il est fabriqué dans les moulins à papier et, de l'autre, parce que ses matériaux - chiffons, funin, etc. - sont eux-mêmes jusqu'au $19^{\mathrm{e}}$ siècle des produits de la civilisation. L'histoire de la technologie du papier, depuis la naissance des premiers moulins à papier européens environ deux cents ans avant l'invention de la presse d'imprimerie jusqu'à l'exploitation de nouvelles sources de matières premières et l'élargissement de la production de papier grâce à la machinerie et à la grande industrie du $19^{\mathrm{e}}$ siècle, constitue le point de départ d'une histoire du papier occidental comme médium. Cette histoire ne devient cependant celle du papier que dans la mesure où elle représente les effets produits par la multiplicité des alliances que cette matière non spécifique contracte avec d'autres médias : en tant que page de livre, papier à lettres, facture ou traite, document estampillé ou bout de papier à notes, découpage ou billet de banque.

La formule consacrée pour signifier l'effet central de l'alliance historique du papier et de la presse d'imprimerie, from manuscript to print, est généralement lue comme une abréviation diachrone du processus par lequel la technologie d'impression du début des Temps modernes remplaça la culture du manuscrit ayant cours au Moyen Âge. C'est pourquoi l'on décrit fréquemment le rôle joué par la presse dans la mise en relation entre textes et auteurs individuels et par là, l'émergence de la notion moderne d'auteur. On ne devient pas auteur seulement pour avoir rédigé des manuscrits, mais parce qu'on fait circuler son œuvre sous forme imprimée. Jusqu'au $17^{\mathrm{e}}$ siècle au plus tard, le concept d'auteur est explicitement lié au processus d'impression dans les dictionnaires². L'impression d'un manuscrit ne se réduit cependant pas au remaniement de la forme d'un texte dans le

2. Voir Uwe Wirth, Die Geburt des Autors aus dem Geist der Herausgeberfiktion. Editoriale Rahmung im Roman um 1800 : Wieland, Goethe, Brentano, Jan Paul und E.T.A. Hoffmann, Munich, Wilhelm Fink, 2008. 
but de produire une multiplicité de copies identiques en circulation dans l'espace et le temps. Elle inclut aussi le passage du texte dans une sphère de révision des erreurs et d'exigence accrue de véracité. Les épreuves et la succession des corrections préliminaires puis définitives font partie de l'impression. L'inévitabilité des erreurs d'impression ne disparaît pas, mais elle est tempérée par la possibilité fondamentale de produire de nouvelles éditions améliorées et révisées. À la suite d'Elizabeth Eisenstein, Niklas Luhmann a interprété cette favorisation du texte le plus récent comme une contribution de la presse d'imprimerie à la valorisation de la nouveauté. Selon lui, la multiplication des textes copiés dans les bureaux de la culture du manuscrit tendait à aller de pair avec un nombre croissant de fautes. Le texte obtenu au terme d'une chaîne de reproduction devait attirer sur lui un soupçon de corruption. Par contraste, l'imprimerie encourageait la supposition selon laquelle la dernière édition d'une œuvre est la meilleure. Et elle dissociait par la même occasion la notion d'«original» d'un texte original dont les copies paraissaient être des produits dérivés. L’ original » devient donc libre de signifier ce qui n'existe pas encore, perdant son caractère d'indicateur du passé pour devenir la forme en creux de la nouveauté3.

La favorisation de la nouveauté ne rejette toutefois pas le passé, car elle l'absorbe en le renouvelant: la presse transforme les manuscrits existants ainsi que les rares exemplaires qui subsistent de leur transmission en nouveautés sur le marché du livre. L'humanisme célèbre la presse non seulement parce qu'elle ressuscite les morts, mais aussi parce qu'elle garantit l'immortalité du savoir. Ceci n'est néanmoins possible que dans la mesure où l'immortalité n'est plus appréhendée comme un effet de la robustesse du support de l'écriture, mais comme celui de la circulation massive du savoir dans des copies identiques. C'est pour cette raison que l'exploitation de la dimension de reproduction par une nouvelle technologie rend caduc l'argument éprouvé dans le cas du papyrus et transposé à la nouvelle constellation, selon lequel les livres imprimés sur papier seraient des médias de conservation incontestablement inférieurs à ceux rédigés ou imprimés sur du parchemin. Dans les propos élogieux qui la mettent en avant, l'imprimerie rend le savoir immortel en le rendant « commun $^{4} »$.

3. Voir Elizabeth L. Eisenstein, The Printing Revolution in Early Modern Europe [1983], Cambridge, Cambridge University Press, 2005, ainsi que Niklas Luhmann, Gesellschaftsstruktur und Semantik. Studien zur Wissenssoziologie der modernen Gesellschaft, vol. 4, Francfort-sur-le-Main, Suhrkamp, 1995.

4. Michael Giesecke, Der Buchdruck in der frühen Neuzeit. Eine historische Fallstudie über die Durchsetzung neuer Informations- und Kommunikationstechnologien [1994], Francfort-sur-le-Main, Suhrkamp, 2006. À propos du scepticisme concernant la durabilité 
L'imprimerie en tant que médium de révision, de reproduction et de durabilité gagne en autorité. Ceci s'entend notamment dans le reproche qui lui est fait de valoriser l'éphémère au lieu de le reléguer dans l'oubli. Ce phénomène a lieu tôt si l'on considère la sphère de l'imprimé dans laquelle le climat de nouveauté de l'époque se condense de manière particulière: la presse périodique. Dans la pièce de Ben Jonson The Staple of News (1625), un des personnages est d'avis que les journaux profitent de l'autorité et de la présomption de valeur qui imprègnerait tout imprimé aux yeux du public: «The very printing of them makes them news, / That ha' not the heart to believe anything / But what they see in print $»^{5}$.

Le scepticisme articulé par le personnage de Jonson n’équivaut pas encore au proverbe «il ment comme l'imprimé », mais il rend déjà palpable le revers qui limite le triomphe de la presse d'imprimerie en tant que machine autorisant la production d'autorité littéraire et d'autorité tout court. La question qui se pose est celle de l'origine de l'imprimé. Lapparition de cette question s'explique par le fait que le marché ne valorise pas seulement la révision constante du savoir sub specie ceternitatis, mais également la multiplication de la nouveauté qui ne subsiste que parce qu'elle est nouvelle. Quiconque voit dans la presse d'imprimerie, à l'instar du personnage de Jonson, l'instance grâce à laquelle les nouveautés sont autorisées en tant que telles n'est pas loin de soupçonner qu'une bonne partie de tout ce qui est imprimé profite à tort de la fonction d'autorisation de la presse d'imprimerie.

From manuscript to print - la formule désigne, dans l'horizon de la notion moderne d'auteur, le transport de la transcription manuelle dans un espace de normalisation et de standardisation où l'exigence de véracité est accrue et l'identité textuelle plus fiable. Elle illustre de plus une supposition fondamentale, à savoir que, depuis l'invention de Gutenberg, la presse d'imprimerie constitue le centre de gravité des processus de transcription derrière lesquels la culture du manuscrit apparaît comme une espèce de sous-traitant défraîchi de l'industrie typographique. La bibliothéconomie récente a cependant indiqué les limites de la thèse selon laquelle l'imprimerie aurait engendré ces effets soudains et profonds que sont la standardisation, l'uniformisation et la normalisation ${ }^{6}$. De

du papier, voir Johannes Trithemius, De laude scriptorum. Zum Lobe der Schreiber, Klaus Arnold (dir.), Würzburg, Freunde mainfränkischer Kunst und Geschichte, 1973.

5. Voir Charles Sommerville, The News Revolution in England: Cultural Dynamics of Daily Information, Oxford \& New York, Oxford University Press, 1996.

6. Voir David McKitterick, Print, Manuscript and the Search for Order. 1450-1830, Cambridge, Cambridge University Press, 2003. 
nombreuses études sur la juxtaposition de la circulation des manuscrits et la diffusion d'imprimés à l'époque des Temps modernes ont parallèlement contredit l'idée que la formule from manuscript to print désignerait - chronologiquement et fonctionnellement - la « relève » de la culture du manuscrit par la presse d'imprimerie ${ }^{7}$. "L'imprimerie resta jusqu'au début du temps de Goethe une branche de production destinée aux élites de la noblesse et de la bourgeoisie, tandis que la majeure partie de la population européenne, si tant est qu'elle soit entrée en contact avec l'écrit, avait presque exclusivement affaire à des écrits rédigés à la main (dossiers et lettres), exception faite des quelques imprimés vraiment populaires tels que les sermons de Luther et le catéchisme ${ }^{8}$. "En d'autres termes, la masse du papier non imprimé devait encore excéder celle du papier imprimé au $19^{\mathrm{e}}$ siècle.

Dans la mesure où la fixation sur le livre imprimé comme astre central supposé du monde de Gutenberg se relâche, la suggestion diachrone qui émane de la formule from manuscript to print fait ressortir la cohabitation fonctionnelle et la tension synchrone qui existent entre le manuscrit, non plus médiéval mais moderne, et l'impression. La prise en compte de l'interdépendance conflictuelle entre «manuscrit» et «impression» est d'une importance capitale pour une théorie du médium papier. Celle-ci permet en effet de mettre les imprimés sur papier en rapport avec la multiplicité des techniques culturelles dans lesquelles le papier porte des inscriptions, est peint, etc., mais pas imprimé. D’autre part, elle permet également d'appréhender la constellation moderne qui émerge avec la presse comme celle d'un face-à-face du papier imprimé et non imprimé. La formule from manuscript to print désigne donc l'horizon optionnel qui entoure, sur le plan synchrone, chaque enregistrement de lettres, chiffres et autres signes - quel que soit le support sur lequel ceux-ci sont couchés - une fois que la presse d'imprimerie voit le jour. Une seconde interprétation de cette formule propose que non seulement l'imprimé, mais aussi le non-imprimé, apparaissent avec la presse d'imprimerie. Ceci peut paraître futile, pourtant c'est d'une importance capitale si l'on veut comprendre la fusion entre la technologie du papier et la presse d'imprimerie. Cela s'explique par le fait que, comme il a déjà été suggéré, le non-imprimé moderne ne s'épuise pas dans la transmission manuscrite du

7. Voir Julia Crick et Alexandra Walsham (dir.), The Uses of Script and Print, 13001700, Cambridge, Cambridge University Press, 2004, et Gerd Dicke et Klaus Grubmüller (dir.), Die Gleichzeitigkeit von Handschrift und Buchdruck, Wiesbaden, Harrassowitz, 2003.

8. Arno Menzel-Reuters, «Das Nebeneinander von Handschrift und Buchdruck im 15. und 16. Jahrhundert» dans Ursula Rautenberg (dir.), Buchwissenschaft in Deutschland. Ein Handbuch, vol. 1, Berlin, De Gruyter Saur, 2010, p. 434. 
Moyen Âge, transmission qui fut entretenue par les humanistes à travers une succession de nouvelles éditions imprimées. Celui-ci s'enracine dans un lent processus en marche depuis le $13^{\mathrm{e}}$ siècle, à savoir le déploiement du papier aussi bien en tant que nouveau médium de transcription qu'en tant que support de l'image. Deux puissants médias fusionnent dans l'union de la presse d'imprimerie et du papier, et la relation entre non-imprimé et imprimé donne naissance à ces oppositions binaires dynamiques qui font avancer le développement culturel du fait de leur potentiel de tension latent.

L'augmentation du prestige culturel du livre et de son instrument, la presse d'imprimerie, est largement documentée. Mais le papier aussi a revendiqué sa place dans la sphère que l'on pourrait nommer l'ordre symbolique de l'«ère Gutenberg». Cette place est obscurcie si l'on appréhende la fusion du papier et de la presse d'imprimerie exclusivement à l'horizon de l'opposition entre oralité et écriture qui remonte à l'Antiquité. Avec ce face-à-face synchrone entre imprimé et non-imprimé s'ouvre en effet, à l'intérieur du processus général de scripturalisation, une deuxième opposition dynamique de structure paradoxale. Le paradoxe résulte de la différence culturelle élémentaire qui s'établit ici à l'intérieur du même médium au lieu d'être partagée entre deux médias différents. Au fur et à mesure que le parchemin et d'autres supports d'écriture du monde moderne sont marginalisés, l'opposition entre imprimé et non-imprimé devient essentiellement une opposition entre papier imprimé et non-imprimé. Alors que l'opposition générale entre écriture et oralité est liée à la différence entre œil et oreille d'un point de vue anthropologique historique et à la différence entre médiation et immédiateté pour la théorie de la communication, une opposition dynamique entre imprimé et non-imprimé s'établit au sein de la sphère que nous appréhendons en la généralisant à l'aide du concept de «culture de l'écrit». Au lieu de disparaître dans le mouvement from manuscript to print, celle-ci accompagne la marche triomphale de la presse d'imprimerie à chaque étape de son développement. Elle établit un ordre symbolique dans lequel l'imprimé est relié à travers un ensemble de signaux à ce monde en arrière-plan que représente le papier non imprimé. Ce dernier occupe ainsi une position paradoxale qui est reflétée de plusieurs manières dans la littérature des Temps modernes. À l'instar des imprimés, le papier soutient bien évidemment la tendance générale à la scripturalisation. Néanmoins, il se trouve aussi dans une position symbolique analogue à celle de l'oralité dans la mesure où le mot imprimé devient le noyau de la sphère publique moderne.

Il suffit de considérer, même rapidement, la poétique du roman épistolaire pour s'apercevoir que la tension n'y est pas moindre entre ce «discours 
vivant» qu'est l'écriture manuelle, proche du corps et couchée sur du papier non imprimé, et l'imprimé. L'apparition du papier dans les conventions d'usage pour les titres de livres et d'articles de revues nous renseigne sur la formation de cette constellation. Un titre tel que Pensées de Mr. Pascal sur la religion, et sur quelques autres sujets, Qui ont esté trouvées aprés sa mort parmy ses papiers, A Amsterdam, Chez Abraham Wolfganck 1677 rattache explicitement le texte publié à son origine non imprimée. Il est typique surtout parce qu'il fait le récit implicite d'une histoire dans laquelle l'éditeur est le personnage principal. D'après la convention du titre, l'auteur décédé est présenté comme un second héros qui fait face à l'éditeur. En fouillant dans les papiers laissés par le défunt et en pratiquant une sélection qui est ensuite mise sous presse, celui-ci élargit l'autorité littéraire de l'auteur. Ainsi l'auteur ne pouvait écrire que du temps de son vivant, mais son autorité ne s'arrête pas au moment de sa mort. Les Pensées seront au centre de l'œuvre de Pascal pendant les siècles suivants. Louverture au futur, en principe infinie, de cette autorité ancrée dans le papier non imprimé est suggérée par l'usage qui consiste à présenter des «papiers inédits » comme une sélection effectuée à partir d'un réservoir qui n’a pas encore été épuisé. Ce n’est pas seulement le chef-d'œuvre d'un auteur qui se dissimule dans ce monde en arrière-plan du papier non imprimé dont le développement a lieu parallèlement à la marche triomphale de l'imprimé, mais aussi une vérité politique ou historique.

Avec l'avènement des journaux au début du $17^{\mathrm{e}}$ siècle, le réservoir du nonimprimé rivalise avec les nouvelles et opinions publiées dans la presse périodique, ainsi qu'avec l'historiographie. C'est pour cette raison que la sphère publique moderne, qui réserve une place à la discussion politique, n'est pas seulement une sphère dans laquelle la vérité est débattue dans un affrontement de voix. Les conjectures qui prétendent dire la vérité se réfèrent aussi bien aux "papiers inédits» qui se trouvent encore dissimulés dans la sphère privée qu’au discours public. Un titre comme The History of the most unfortunate Prince, King Edward II with Choice Political Observations on Him and his unhappy Favourites, Gaveston \& Spencer, Found among the Papers of, and (supposed to be) Writ by the Right Honourable Henry Viscount Faulkland, London 1680 peut concéder que l'origine auctoriale des papiers découverts est incertaine, sans que la promesse de révision et de complémentation des vérités imprimées soit compromise. En effet, lorsque l'ordre symbolique de l'imprimé est établi comme source de vérité, la presse d'imprimerie ratifie l'autorité des unpublished papers plus qu'elle ne la produit d'elle-même à travers l'impression.

Voilà pourquoi les effets historiques obtenus à l'interface de l'impression ne peuvent être que partiellement décrits à partir du point de vue seul de la 
presse d'imprimerie. Ils n'apparaissent pas seulement à la suite de l'intégration du non-imprimé dans la sphère de l'imprimé conformément à la logique propre à la presse d'imprimerie. Ils émergent de la fusion de la sphère des "papiers imprimés ", qui est dotée d'une forte logique et d'une dynamique propre, avec son contraire. Marshall McLuhan a conçu son image de la galaxie Gutenberg en en axant la perspective centrale sur la presse d'imprimerie. Malgré sa propension à la dramatisation de la théorie des médias, il est parvenu, dans ses réflexions sur l'«énergie issue des hybrides", à une vue que l'on peut rendre productive pour écrire l'histoire de l'opposition dynamique entre imprimé et non-imprimé. Il y compare en effet les forces et énergies qui sont libérées par «croisement ou hybridation des médias» avec les effets de la «scission ou fusion nucléaire ${ }^{9}$ ».

Le passage des «papiers inédits» dans la sphère du papier imprimé sanctionne l'autorité du non-imprimé et abolit par la même occasion son statut d'agrégat. Le passage du papier non imprimé dans les conventions régissant les titres constitue le résidu de cette fusion. La stratégie de publication adoptée par Gotthold Ephraim Lessing dans le Fragmentenstreit (Querelle des fragments) en est un exemple célèbre. Le titre choisi pour la première livraison Von Duldung der Deisten: Fragment eines Ungenannten (Tolérance envers les déistes: Fragments tirés des écrits d'un auteur anonyme, 1774) établit une figure de l'auteur qui est redéfinie par le monde en arrière-plan dans lequel Lessing œuvre en tant qu'éditeur pour la deuxième livraison: Ein Mehreres aus den Papieren des Ungenannten, die Offenbarung betreffend (Un supplément tiré des papiers de l'auteur anonyme concernant la révélation, 1777). Le titre était un instrument idéal pour mettre de l'huile sur le feu du questionnement touchant à la vérité de la tradition biblique. L'auteur était en effet déjà anonyme lors de sa première apparition et il le resta ostensiblement pour la seconde car il devait être protégé post-mortem de l'explosivité de ses écrits. Lauteur anonyme est cette figure auctoriale dans laquelle les frontières du discours officiel se dessinent. C'est un auteur publié qui demeure en même temps dans la sphère du papier non imprimé. Un paradoxe s'introduit dans sa voix publique: celle-ci dit quelque chose dans la sphère du mot imprimé dont l'énonciation ne peut pas être attribuée avec certitude. Lessing, qui était à la fois défenseur et éditeur de cette voix paradoxale, s'attira une interdiction de publier qui mit un terme à la querelle des fragments.

«Found Among the Papers... ", «trouvés parmi les papiers de...», «aus den Papieren... » : la promesse - ou menace - d'un «à suivre» se dissimule dans des 
prépositions en apparence insignifiantes. Quand pendant la Révolution française, après Thermidor, Edme-Bonaventure Courtois rendit publique la vérité sur Robespierre et sa clique sous le titre Rapport fait au nom de la commission chargée de l'examen des papiers trouvés chez Robespierre et ses complices dans la séance du 16 nivôse, an III. De la République française, Paris, Impr. Nat. Des lois, l'an III (1795), ce rapport de commission pouvait être sûr d'être suivi d'un écho qui se réclamait de l'autorité des papiers omis par Courtois : Papiers inédits trouvés chez Robespierre, Saint-Just, Payan etc., supprimés ou omis par Courtois; Précédés du Rapport de ce député à la Convention Nationale, avec un grand nombre de facsimile et les signatures de principaux personnages de la Révolution. Paris, Baudouin Frères, 1828. La facsimilation de manuscrits et d'autographes, reprise ici explicitement dans le titre et promue par la lithographie au début du $19^{\mathrm{e}}$ siècle, appartient aux techniques permettant de reconnaître son autorité au non-imprimé à travers l'imprimé. Avant que la facsimilation ne devienne omniprésente en tant que procédé technique, la fiction de l'éditeur lui correspondait depuis longtemps sur le plan poétologique dans la littérature des Temps modernes. Déjà dans Don Quichotte, cette fiction ne se limite pas à fournir un récit des origines faisant le lien entre le livre imprimé et le manuscrit non imprimé. Elle établit plutôt un axe généalogique actualisable à tout moment entre le texte imprimé et le manuscrit dont celui-ci est issu. Elle s'efforce de rendre la feuille du livre transparente dans l'imagination du lecteur et de suggérer la présence virtuelle du non-imprimé dans l'imprimé. C'est pourquoi Don Quichotte n'est pas qu'un exemple classique des ensembles thématiques du livre dans le livre ainsi que du lecteur dans la bibliothèque, mais il illustre aussi l'ancrage suggestif de la fiction de l'éditeur dans le monde en arrière-plan du papier non relié.

Dans Don Quichotte, la plume de l'auteur arabe du manuscrit, Cide Hamete Benengeli, a le dernier mot et pour plusieurs raisons. Ceci non seulement pour clore le jeu capricieux avec le lecteur que le narrateur de Cervantès entame à partir de la visite du marché de soie et de papier à Tolède et de l'introduction de l'auteur arabe dans le neuvième chapitre de la première partie; mais surtout pour éliminer ce concurrent anonyme, pourtant bien réel, qui kidnappe ce titre ainsi que ses personnages romanesques à l'auteur Cervantès en 1614, un an avant la parution de la deuxième partie de Don Quichotte, pour les incorporer à son propre roman et présenter ce dernier comme la suite légitime de la première partie de l'œuvre de Cervantès sur le marché. C'est seulement une fois la plume de Cide Hamete pendue à son clou, et non au moment de la mort de Don Quichotte, que Cervantès met un terme définitif au roman, excluant toute 
possibilité de suite. Il ratifie de sa propre main droite valide le discours de Cide Hamete dans lequel celui-ci renonce à son statut d'auteur :

Pour moi seul naquit Don Quichotte, et moi pour lui. Il sut agir, et moi écrire. Nous deux seuls ne faisons qu'un, en dépit et à la barbe de l'écrivain imposteur et tordesillesque qui osa, ou qui va oser écrire d'une plume d'autruche grossière et mal taillée les exploits de mon valeureux chevalier. Ce n'est pas une charge pour ses épaules, ni un sujet pour son froid génie ${ }^{10}$.

Lorsque le lecteur est arrivé à cet endroit qui retransforme virtuellement le roman imprimé qu'il a sous les yeux en une écriture manuelle non imprimée, Don Quichotte et lui ont déjà visité une imprimerie à Barcelone dans laquelle un livre est lui aussi en train d'être corrigé parmi tant d'autres: «comme il demandait le titre, on lui répondit qu'il s'appelait La Seconde Partie de l'Ingénieux Hidalgo Don Quichotte de la Manche, composée par un certain habitant de Tordesillas $^{11} »$. Cette rencontre de Don Quichotte avec le livre que son double a mis au monde constitue l'équivalent des scènes situées dans la deuxième partie du roman dans lesquelles Don Quichotte et Sancho Panza rencontrent le lecteur de la première partie. Don Quichotte n'est en effet pas seulement l'apothéose du typographic man, du lecteur qui se perd dans sa bibliothèque de romans chevaleresques. C'est également une apothéose de la double nature de l'auteur moderne en laquelle fusionnent les énergies issues de la tension entre papier imprimé et papier non imprimé. C'est à partir de cette tension que le support matériel de la littérature des Temps modernes pénétra dans le cercle de la matière poétique de celle-ci. Sur ses formes matérielles, sur la feuille et le bout de papier, sur le papier jauni des chancelleries et le papier à lettres imbibé de larmes, etc., se fixent des histoires, des métaphores et des motifs dans lesquels la littérature s'auto-réfléchit.

Traduit de l'allemand par Hélène Sicard-Cowan

10. Miguel de Cervantes Saavedra, Don Quichotte, Jean-Raymond Fanlo (ed. et trad.), Paris, LGF/Livre de poche, 2008, p. 706.

11. Ibid., p. 612. 
